J Comput Theor Nanosci. 2009 September 1; 6(9): 1979-1992. doi:10.1166/jctn.2009.1254.

\title{
Photonic Nanojets
}

\author{
Alexander Heifetz ${ }^{a,{ }^{*}, \text { Soon-Cheol Kong }}{ }^{b,{ }^{* *} ;,{ }^{*}, \text { Alan V. Sahakian }}{ }^{a, b}$, Allen Taflove ${ }^{b}$, and Vadim \\ Backman ${ }^{\mathrm{a}}$ \\ a Department of Biomedical Engineering, Northwestern University, Evanston, IL 60208 USA \\ $\mathrm{b}$ Department of Electrical Engineering and Computer Science, Northwestern University, Evanston, \\ IL 60208 USA
}

\begin{abstract}
This paper reviews the substantial body of literature emerging since 2004 concerning photonic nanojets. The photonic nanojet is a narrow, high-intensity, non-evanescent light beam that can propagate over a distance longer than the wavelength $\lambda$ after emerging from the shadow-side surface of an illuminated lossless dielectric microcylinder or microsphere of diameter larger than $\lambda$. The nanojet's minimum beamwidth can be smaller than the classical diffraction limit, in fact as small as $\sim \lambda / 3$ for microspheres. It is a nonresonant phenomenon appearing for a wide range of diameters of the microcylinder or microsphere if the refractive index contrast relative to the background is less than about 2:1. Importantly, inserting within a nanojet a nanoparticle of diameter $d_{v}$ perturbs the farfield backscattered power of the illuminated microsphere by an amount that varies as $d_{v}{ }^{3}$ for a fixed $\lambda$. This perturbation is much slower than the $d_{v}{ }^{6}$ dependence of Rayleigh scattering for the same nanoparticle, if isolated. This leads to a situation where, for example, the measured far-field backscattered power of a 3- $\mu \mathrm{m}$ diameter microsphere could double if a 30-nm diameter nanoparticle were inserted into the nanojet emerging from the microsphere, despite the nanoparticle having only $1 / 10,000^{\text {th }}$ the cross-section area of the microsphere. In effect, the nanojet serves to project the presence of the nanoparticle to the far field. These properties combine to afford potentially important applications of photonic nanojets for detecting and manipulating nanoscale objects, subdiffractionresolution nanopatterning and nanolithography, low-loss waveguiding, and ultrahigh-density optical storage.
\end{abstract}

\section{Introduction}

Since 2004, a substantial literature ${ }^{1-20}$ has developed regarding the existence, properties, and potential applications of the photonic nanojet. The photonic nanojet is a narrow, high-intensity electromagnetic beam that propagates into the background medium from the shadow-side surface of a plane-wave illuminated lossless dielectric microcylinder or microsphere of diameter greater than the illuminating wavelength, $\lambda$. Key properties of the photonic nanojet include: (1) It is a non-evanescent, propagating beam that can maintain a subwavelength fullwidth at half-maximum (FWHM) transverse beamwidth along a path that can extend more than $\sim 2 \lambda$ beyond the dielectric cylinder or sphere. (2) Its minimum FWHM beamwidth can be smaller than the classical diffraction limit, in fact as small as $\sim \lambda / 3$ for microspheres. (3) It is a nonresonant phenomenon that can appear for a wide range of the diameter $d_{\mu}$ of the dielectric microcylinder or microsphere from $\sim 2 \lambda$ to more than $40 \lambda$ if the refractive index contrast relative to the background medium is less than about 2:1. (4) It has a high intensity that can significantly exceed that of the illuminating wave. (5) Inserting within a nanojet a nanoparticle of diameter

**sch.kong@gmail.com.

*Co-principal author. 
$d_{v}$ perturbs the far-field backscattered power of the illuminated microsphere by an amount that varies as $d_{v}{ }^{3}$ for a fixed $\lambda$. This perturbation is much slower than the $d_{v}{ }^{6}$ dependence of Rayleigh scattering for the same nanoparticle, if isolated. This leads to a situation where, for example, the measured far-field backscattered power of a $3-\mu \mathrm{m}$ diameter microsphere could double if a 30-nm diameter nanoparticle were inserted into the nanojet emerging from the microsphere, despite the nanoparticle having only $1 / 10,000^{\text {th }}$ the cross-section area of the microsphere. In effect, the nanojet serves to project the presence of the nanoparticle to the far field.

These properties combine to afford potentially important applications of photonic nanojets for detecting and manipulating nanoscale objects, subdiffraction-resolution nanopatterning and nanolithography, low-loss waveguiding, and ultrahigh-density optical storage. With the aim of encouraging nanojet research, this paper summarizes progress in the nanojet area to-date. Discussions include the initial identification of the photonic nanojet for a dielectric cylinder (Section II); the initial identification of the photonic nanojet for a dielectric sphere (Section III); photonic nanojet theory (Section IV); laboratory observations (Section V); and potential applications (Section VI).

\section{Initial Identification of the Photonic Nanojet for a Dielectric Cylinder}

The initial identification of the photonic nanojet as a distinct electromagnetic wave type and the coining of the descriptor "photonic nanojet" was reported by Chen et al. ${ }^{1}$ Applying a highresolution finite-difference time-domain (FDTD) computational solution of Maxwell's equations, ${ }^{21}$ evidence was provided that a plane-wave illuminated, micron-scale, circular dielectric cylinder can generate a narrow, high-intensity, subdiffraction-waist beam that propagates into the background medium from the cylinder's shadow-side surface. Furthermore, it was reported that positioning a nanometer-scale dielectric cylinder within the nanojet causes a giant perturbation of the backscattered power of the micron-scale dielectric cylinder emitting the nanojet. That is, the perturbation is orders of magnitude larger than the backscattering of the nanoscale cylinder when considered in isolation.

Reference 1 first reviewed the validation of its FDTD computational technique, which employed a uniform square cell size of $1.25 \mathrm{~nm}$ (finer than 1/100th dielectric wavelength for all modeling runs) and the perfectly matched layer absorbing outer grid boundary condition.

${ }^{21}$ Upon comparing the FDTD-computed differential scattering cross-section of several homogeneous, isotropic, circular dielectric cylinders with the exact series solution, agreement was found to within $\pm 1 \mathrm{~dB}$ over the entire range of scattering angles for all cases studied over dynamic ranges of $60 \mathrm{~dB}$.

Reference 1 then reported detailed two-dimensional computational studies of the internal and near-external fields of a series of homogeneous, isotropic, lossless, infinitely long, circular dielectric cylinders. Each cylinder was assumed to be illuminated by a plane wave polarized with the incident electric field parallel to the cylinder's infinite axis. Figure 1 shows sample results illustrating the evolution of a photonic nanojet as the refractive index of a circular dielectric cylinder decreases. Here, the FDTD-computed envelope of the sinusoidal steadystate electric field is visualized for a $d_{\mu}=5 \mu \mathrm{m}$ diameter circular cylinder of uniform refractive index $n_{1}$ embedded within an infinite vacuum medium of refractive index $n_{2}=1$.0. Light of wavelength $\lambda_{2}=500 \mathrm{~nm}$ propagates from left to right in medium 2 . Upon decreasing $n_{1}$ from 3.5 in Fig. 1(a) to 2.5 in Fig. 1(b), the internal electric-field peak shifts toward the shadow-side surface of the cylinder. Upon further decreasing $n_{1}$ to 1.7 in Fig. 1(c), the electric-field peak emerges from the shadow-side surface of the cylinder as a strong jet-like distribution having a length of $\sim 900 \mathrm{~nm}\left(1.8 \lambda_{2}\right)$ and a weakly subdiffraction FWHM intensity waist of $\sim 200 \mathrm{~nm}$ $\left(0.4 \lambda_{2}\right)$. The emergence of this jet from the shadow-side surface of the cylinder for $n_{1} \sim 2$ is consistent with previous work on optical caustics. ${ }^{22,} 23$ 
Reference 1 then reported that weakly subdiffraction-waist photonic nanojets similar to that in Fig. 1(c) can be generated using a variety of combinations of $d_{\mu}, n_{1}, n_{2}$, and $\lambda_{2}$, provided that $n_{1} / n_{2}$ is not significantly changed from that of Fig. 1(c). Examples are illustrated in Fig. 2 for (a) $d_{\mu}=5 \mu \mathrm{m}, n_{1}=3.5, n_{2}=2.0, \lambda_{2}=250 \mathrm{~nm}$; (b) $d_{\mu}=6 \mu \mathrm{m}, n_{1}=2.3275, n_{2}=1.33, \lambda_{2}=300$ $\mathrm{nm}$; and (c) $d_{\mu}=10 \mu \mathrm{m}, n_{1}=2.3275, n_{2}=1.33, \lambda_{2}=300 \mathrm{~nm}$. Including the case of Fig. 1(c), the FWHM intensity waists of all nanojets modeled ranged between $0.4 \lambda_{2}$ and $0.48 \lambda_{2}$, and the lengths of all nanojets modeled ranged between $1.6 \lambda_{2}$ and $2 \lambda_{2}$.

Finally, using FDTD modeling, Reference 1 considered the impact of positioning a nanometerscale perturbing dielectric cylinder within the nanojet generated by a micron-scale primary cylinder. The test case used the parameters of Fig. 2(b), i.e., a $6 \mu \mathrm{m}$ diameter primary circular cylinder of refractive index $n_{1}=2.3275$ embedded within an infinite exterior medium of refractive index $n_{2}=1.33$, and illuminated at the wavelength $\lambda_{2}=300 \mathrm{~nm}$. The perturbing dielectric nanocylinder was assumed to have a square cross-section of side dimension $d_{v}=5$ $\mathrm{nm}$ and refractive index $n_{v}=1.5$. This nanocylinder was inserted in the FDTD model at the center of the photonic nanojet on the surface of the $6 \mu \mathrm{m}$ diameter primary cylinder.

Figure 3(a) graphs the absolute value of the resulting perturbation of the differential scattering cross-section of the primary microcylinder within $\pm 10^{\circ}$ of backscatter. This figure also graphs the corresponding differential scattering cross-section of the isolated, plane-wave illuminated perturbing nanocylinder. These results show that the perturbation of the direct backscattering cross-section of the microcylinder provided by the 5-nm dielectric nanocylinder located within the nanojet is approximately $4.5 \times 10^{4}$-times greater than the backscattering of the isolated nanocylinder. Figure 3(b) illustrates the results of additional FDTD modeling studies in Reference 1 which show that this effective enhancement of the backscattering of the perturbing nanocylinder, designated as $E$, increases monotonically as $d_{v}$ decreases below $20 \mathrm{~nm}$, reaching a level of approximately $10^{6}$ for $d_{v}=1.25 \mathrm{~nm}$.

As a result of these computational modeling studies, it was concluded in Reference 1 that photonic nanojets may provide a new means to detect nanoparticles of size well below the classical diffraction limit. This could potentially yield means to use visible light to detect proteins, viral particles, and even single molecules; and to monitor molecular synthesis and aggregation processes of importance in many areas of biology, chemistry, materials sciences, and tissue engineering.

\section{Initial Identification of the Photonic Nanojet for a Dielectric Sphere}

The initial identification of the photonic nanojet for a dielectric sphere was reported by $\mathrm{Li}$ et $\mathrm{al}^{2}$ Their approach involved implementing an exact eigenfunction series solution of Maxwell's equations in spherical coordinates (i.e., Mie theory) to compute the near field external to each plane-wave illuminated dielectric sphere of interest. In this manner, evidence was provided that a plane-wave illuminated, micron-scale dielectric sphere can generate a fully threedimensional photonic nanojet having a weakly subdiffraction minimum beamwidth. This nanojet has properties that are similar to its two-dimensional counterpart produced by a planewave illuminated circular dielectric cylinder of comparable size and refractive index contrast relative to the background medium. However, the nanojet generated by the dielectric sphere has an even higher intensity than the nanojet generated by the dielectric cylinder. Furthermore, the backscattered power of the dielectric sphere is perturbed to an even greater degree than that of the dielectric cylinder when a nanometer-scale particle is positioned within the nanojet.

Referring to Fig. 4, Reference 2 first studied a series of homogeneous, isotropic, dielectric microspheres of refractive index $n=1.59$. Each microsphere was assumed to be illuminated in an infinite vacuum region by an $x$-polarized, $z$-propagating plane wave with unit intensity and wavelength $\lambda=400 \mathrm{~nm}$. Figures 4(a), 4(b), 4(c), and 4(d) visualize the computed near- 
external field of each microsphere for diameters $d_{\mu}=1 \mu \mathrm{m}, 2 \mu \mathrm{m}, 3.5 \mu \mathrm{m}$, and $8 \mu \mathrm{m}$, respectively. For $d_{\mu}$ less than $4 \mu \mathrm{m}$ (Figs. 4(a) - (c)), a weakly subdiffraction-waist nanojet protrudes from the shadow side of the sphere. For larger $d_{\mu}$ (Fig. 4(d)), the intensity peak of the nanojet moves away from the microsphere's surface. Also noted is that both the maximum intensity and FWHM waist of the nanojet increase as $d_{\mu}$ increases.

Reference 2 then studied the magnitude of the perturbation of the backscattered power of the $d_{\mu}=3.5 \mu \mathrm{m}$ microsphere of Fig. 4(c) caused by the passage of a $d_{v}=20 \mathrm{~nm}$ gold nanoparticle of refractive index $n_{v}=1.47-\mathrm{j} 1.95$ through the nanojet generated by microsphere. We shall designate the peak backscattered power perturbation magnitude caused by the gold nanoparticle as $|\delta|$. Figure 5(a), which shows all particle dimensions to-scale, illustrates how the gold nanoparticle was assumed to travel in the $y$-direction along a straight-line path in the vacuum located $240 \mathrm{~nm}$ from the shadow-side surface of the primary microsphere. The backscattering of the composite microsphere/gold nanoparticle system was computed using the generalized multiparticle Mie (GMM) technique, which provides a rigorous analytical solution for electromagnetic wave scattering by multiple spheres based upon the addition theorems for vector spherical wave functions. ${ }^{24,25}$

Referring to Fig. 5(b), it was determined that the $d_{v}=20 \mathrm{~nm}$ gold nanoparticle moving along the path illustrated in Fig. 5(a) causes a peak backscattered power perturbation $|\delta|=40 \%$ relative to the backscattered power of the isolated microsphere. This is a relatively giant perturbation, only $4 \mathrm{~dB}$ below the full backscattered power of the isolated microsphere, that is caused by a nanoparticle fully 175 -times smaller than the microsphere in diameter (i.e., more than 30,000times smaller than the microsphere in cross-section area).

Referring to Fig. 5(c), the above study was repeated for gold nanoparticles ranging in diameter from $d_{v}=2 \mathrm{~nm}$ to $d_{v}=60 \mathrm{~nm}$. It was determined that $|\delta|$ increases monotonically over this size range, in fact exceeding $100 \%$ of the backscattered power of the isolated microsphere for gold nanoparticles as small as $d_{v}=27 \mathrm{~nm}\left(\sim 1 / 130^{\text {th }}\right.$ of the diameter of the microsphere).

Interestingly, a $d_{v}=2 \mathrm{~nm}$ gold particle, i.e., a clump of about 100 gold atoms, was found to generate a $|\delta|$ just $34 \mathrm{~dB}$ below the full backscattered power of the isolated microsphere. Consequently, instruments having a dynamic range of $40 \mathrm{~dB}$ or more could detect such near atomic-level size particles using visible light.

Furthermore, Fig. 5(c) reveals that the backscattered power perturbation $|\delta|$ caused by the gold nanoparticle increases as $d_{v}^{3}$ over the nanoparticle diameter range $2 \mathrm{~nm} \leq d_{v} \leq 20 \mathrm{~nm}$ (i.e., $\lambda /$ $200 \leq d_{v} \leq \lambda / 20$ ). This is a much slower than the $d_{v}{ }^{6}$ dependence of Rayleigh scattering.

Reference 2 proceeded to calculate for each gold nanoparticle diameter the effective backscattering enhancement factor $E$ provided by the photonic nanojet. $E$ was defined as $|\delta|$ normalized by the backscattered power of the isolated, plane-wave illuminated gold nanoparticle. Referring to Fig. 6(a), it was determined that $E$ rises from approximately $3 \times$ $10^{4}$ to $2 \times 10^{8}$ as the size of the gold nanoparticle drops from $60 \mathrm{~nm}$ to $2 \mathrm{~nm}$. These values are, respectively, 30-times larger and 200-times larger than the corresponding enhancement factors for the two-dimensional cylinder nanojet case shown in Fig. 3(b). For nanoparticle diameters below $20 \mathrm{~nm}$, this increase in $E$ takes on a $d_{v}^{-3}$ dependence. In combination with the appreciable values of $|\delta|$ in this size range (Fig. 5(c)), it becomes possible to distinguish between gold nanoparticles having diameters differing by as little as $1 \mathrm{~nm}$, as shown in Fig. 6(b).

\section{Photonic Nanojet Theory}

Lecler et al. ${ }^{3}$ applied Mie theory to analyze the general three-dimensional vectorial properties of photonic nanojets generated by plane-wave illuminated dielectric microspheres in free space. They reported that nanojets can be generated for a wide range of microsphere diameters 
$d_{\mu}$ from $\sim 2 \lambda$ to greater than $40 \lambda$. They further reported that the sub-wavelength waist of the nanojet is a consequence of the proximity of the microsphere's shadow-side surface and its focus point in the exterior region (with the microsphere considered as a lens). Specifically, for the microspheres considered, it was found that the nanojet waist remains sub-wavelength for a maximum propagation distance of $\sim 2 \lambda$ for an optimum microsphere refractive index $n_{\mu} \sim$ 1.3. Finally, nanojets were shown to have the same electric-field polarization as the incident plane wave, but with an asymmetric beamwidth (narrower in the direction of the incident magnetic field).

Itagi and Challener ${ }^{4}$ provided a detailed analysis of the optics of photonic nanojets in two dimensions for the case of a plane-wave illuminated, infinitely long dielectric cylinder. Their starting point was an eigenfunction solution of the Helmholtz equation which was recast into a Debye series (an infinite sum of inward and outward radially propagating cylindrical wave modes, each of which can undergo reflection and transmission at the cylinder surface). It was shown that the first term of the Debye series is of particular importance. This term yields a compact expression which connects the physical and geometrical optics properties of the nanojet and permits a straightforward field analysis. Overall, nanojet characteristics were concluded to arise from a "unique combination of features" in the angular spectrum involving the phase distribution and the finite content of propagating and evanescent spatial frequencies.

Chen et al..$^{5}$ reported a perturbation analysis based upon GMM theory for the microsphere/ nanoparticle system to examine the mathematical origins of the $|\delta| \sim d_{v}{ }^{3}$ and $E \sim d_{v}{ }^{-3}$ variations calculated in Reference 2. It was stated that the lens focusing effect of the incident illumination by the dielectric microsphere can account for at most three of the maximum eight orders of magnitude of the backscattering enhancement factor $E$ shown in Fig. 6(a). Furthermore, because the lens focusing effect is constant over the $d_{v}$ range considered, it contributes nothing to the calculated variations of $|\delta|$ and $E$ with $d_{v}$. The physical origins of the $|\delta| \sim d_{v}{ }^{3}$ and $E \sim$ $d_{v}^{-3}$ variations remained unexplained.

Devilez et al. ${ }^{20}$ studied the spatial and spectral properties of the three-dimensional photonic nanojet in a framework employing rigorous Lorentz-Mie theory. They quantitatively evaluated the contributions from all spatial frequency components, both propagating and evanescent. While their study indicated predominantly propagating-wave contributions to the nanojet, the evanescent field contributions created by the illuminated microsphere enhance and sharpen the nanojet's field distribution. A key conclusion of this analysis was that the angular openings of photonic nanojets are at least twice as small as those in comparable Gaussian beams.

\section{Laboratory Observations}

Heifetz et al. ${ }^{6}$ reported an experimental confirmation of the photonic jet for a dielectric sphere and its associated giant backscattering perturbation phenomenon, previously predicted in References 1 and $^{2}$. To facilitate the measurements, the originally reported diameter of the dielectric sphere was scaled upward from the micron scale illuminated by visible light to the centimeter scale illuminated by microwaves at $30 \mathrm{GHz}(\lambda=1 \mathrm{~cm})$. Specifically, the dielectric sphere had a diameter of $7.62 \mathrm{~cm}(7.62 \lambda)$ and was comprised of an acrylic material (polymethylmethacrylate) having the refractive index $n_{\mu}=1.60+\mathrm{j} 0.0016$.

Figure 7(a) depicts the measured data of Reference 6 for the backscattering perturbation $\delta$ caused by a $d_{v}=1 \mathrm{~mm}(\lambda / 10)$ metal particle scanned along the central propagation axis of the microwave jet. Here, $z$ is the distance between the shadow-side surface of the acrylic sphere and the surface of the metal particle. There is good agreement between the measured and FDTD-computed datasets which show that $\delta$ oscillates between positive and negative values depending upon $z$. The perturbation is "giant" in the sense that the 1-mm metal particle nearly doubles the backscattered power of the acrylic sphere if located at $z=2 \mathrm{~mm}, 10 \mathrm{~mm}$, and 15 
$\mathrm{mm}$; and nearly cuts in half the backscattered power of the acrylic sphere if located at $z=6$ $\mathrm{mm}, 12 \mathrm{~mm}$, and $18 \mathrm{~mm}$. This is despite the 1-mm metal particle having a diameter 76-times smaller than the acrylic sphere (i.e., an area almost 6,000-times smaller).

Figure 7(b) shows results analogous to Fig. 7(a), but for the 1-mm metal particle scanned laterally across the microwave jet in the electric-field direction along a line at $z=9 \mathrm{~mm}$ $(0.9 \lambda)$ from the shadow-side surface of the acrylic sphere. There is again good agreement between the measured and FDTD-computed datasets.

Figure 8 depicts the measured data of Reference 6 for the dependence of the backscattered power perturbation $|\delta|$ upon the diameter $d_{v}$ of a perturbing metal particle that is located on the central propagation axis of the microwave jet at $z=9 \mathrm{~mm}(0.9 \lambda)$. Here, the slope of the straightline fit to the measured data yields the dependence $|\delta| \sim d_{v}{ }^{3.34}$ over the range of metal particle diameters $1 \mathrm{~mm} \leq d_{v} \leq 3 \mathrm{~mm}$ (equivalently $0.1 \lambda \leq d_{v} \leq 0.3 \lambda$ ). This is consistent with the theoretical dependence graphed in Fig. 5(b) obtained using the GMM technique for perturbing nanoparticles with diameters $d_{v} \geq 40 \mathrm{~nm}$ (equivalently $d_{v} \geq 0.1 \lambda$ because of the $\lambda=400 \mathrm{~nm}$ illumination wavelength assumed in Fig. 5(b)).

Ferrand et al. ${ }^{13}$ reported the direct experimental observation of photonic nanojets at an optical wavelength, as illustrated in Fig. 9. Here, nanojets were generated by latex microspheres (refractive index $=1.6$ ) of $1 \mu \mathrm{m}, 3 \mu \mathrm{m}$, and $5 \mu \mathrm{m}$ diameters deposited on a glass coverslip and illuminated by a plane wave at $\lambda=520 \mathrm{~nm}$. Measurements were performed using a fast scanning confocal microscope in the detection mode. Each fully three-dimensional photonic nanojet was reconstructed from a collected stack of images which had been corrected by a numerical deconvolution procedure to account for the detection point spread function of the system. Measurements indicated nanojet beamwidths as small as $270 \mathrm{~nm}$ FWHM for a 3- $\mu \mathrm{m}$ diameter microsphere, with the nanojets maintaining sub- $\lambda$ FWHM beamwidths over propagation distances of more than $3 \lambda$.

\section{Potential Applications}

\section{Low-Loss Optical Waveguiding}

Astratov ${ }^{7}, 15$ reported experimental observations of the formation and propagation of nanojetinduced modes (NIMs) in linear chains of several tens of touching polystyrene microspheres (refractive index $n=1.59$ ) with mean diameters in the $2-10 \mu \mathrm{m}$ range. At long distances from the source, measured propagation losses for NIMs were as low as $0.08 \mathrm{~dB}$ per microsphere within a chain formed by $5-\mu \mathrm{m}$ diameter microspheres.

Optical waveguiding in chains of microspheres via NIMs may be advantageous relative to optical waveguiding via intersphere coupling of whispering gallery modes since NIMs are not resonant modes and are inherently broadband. With NIMs, variations of microsphere diameters within a long chain due to normal fabrication tolerances have little or no impact upon the waveguiding characteristics.

\section{Enhanced Raman Scattering}

Yi et al. ${ }^{8}$ reported an experimental observation of enhanced Raman scattering by selfassembled silica microspheres $(1.0 \mu \mathrm{m}$ diameter, refractive index $n=1.5)$. They concluded that the presence of strongly localized optical electric fields within photonic nanojets associated with the silica microspheres accounts for the enhanced Raman scattering.

Microsphere-enhanced Raman spectroscopy may be advantageous relative to conventional surface-enhanced Raman scattering (SERS) approaches because ${ }^{8}$ : (1) the location and size of the dielectric microspheres can be well controlled, eliminating problems with reproducibility 
in SERS; (2) sample preparation is much simpler; and (3) enhanced effects by microspheres can occur for most Raman-active substrates such as silicon. The technique has potential applications in areas of surface science such as oxidation, adhesion, corrosion, and catalytic processes.

\section{Two-Photon Fluorescence Enhancement}

Lecler et al. ${ }^{9}$ reported an experimental observation of the nonlinear enhancement of two-photon excited fluorescence from a molecular dye solution brought about by adding drops of a suspension of silica microspheres $(0.59 \pm 0.1 \mu \mathrm{m}$ diameter, refractive index $n=1.5)$. They argued that the nonlinear absorption is enhanced due to photonic nanojets associated with the microspheres, and suggested that photonic jets could be used to enhance other nonlinear optical effects.

\section{Maskless Subwavelength-Resolution Direct-Write Nanopatterning}

$\mathrm{Wu}$ et al. ${ }^{10}$ reported an experimental demonstration of a maskless subwavelength-resolution direct-write nanopatterning technique which uses a self-assembled planar array of transparent silica or polystyrene microspheres $(0.97 \mu \mathrm{m}$ diameter) deposited on top of a photoresist in a single layer. Upon a brief $(0.8 \mathrm{~s})$ illumination at $\lambda=400 \mathrm{~nm}$, the photoresist was not exposed except at the locations of the high-intensity photonic nanojets associated with the microspheres. Over a large photoresist area, this yielded a regular $0.97 \mu \mathrm{m}$ period array of $250 \mathrm{~nm}$ diameter holes (or pillars if using negative resist processing.) It was determined that this technique provides accurate control of the hole or pillar diameter by varying the exposure time. In addition, the array period could be accurately and independently controlled by using different microsphere sizes. Furthermore, because the nanojet waist is only a weak function of the microsphere diameter, an extremely uniform array of hole or pillar sizes could be achieved even for relatively poor microsphere size uniformity.

McLeod and Arnold ${ }^{16}$ reported the direct writing of complex nanopatterns on a polyimide film substrate using optically trapped microspheres. Here, a $\lambda=532 \mathrm{~nm}$ continuous-wave laser generating a Bessel beam served to optically trap and manipulate a polystyrene or silica microsphere. The trapped microsphere was simultaneously illuminated with a $\lambda=355 \mathrm{~nm}$ pulsed Gaussian beam to generate the nanojet which locally modified the substrate. Optimal results were achieved using $0.76-\mu \mathrm{m}$ polystyrene microspheres. In this manner, arbitrary patterns and individual features with minimum sizes of $\sim 100 \mathrm{~nm}$ (less than one-third of the writing wavelength) were demonstrated. A positioning accuracy of better than $40 \mathrm{~nm}$ was attained in aqueous and chemical environments, and submicron spacing between the microsphere and the substrate was maintained without active feedback control.

\section{Nanoparticle Detection, Sizing, and Location}

As reviewed in Section III, using the GMM analytical technique, Reference 2 first reported the possibility of detecting and sizing gold nanospheres having diameters as small as $2 \mathrm{~nm}$ by exploiting the giant backscattering perturbation phenomenon of the photonic nanojet generated by a dielectric microsphere. Subsequently, Reference 11 applied the GMM technique to determine that, using the photonic nanojet, a 20-nm diameter gold nanosphere could be located with a subdiffraction transverse spatial resolution in the magnetic-field direction as fine as $\lambda /$ 3 in the backgound medium when the nanosphere is approximately $\lambda / 4$ behind the dielectric microsphere that originates the nanojet. The backscattering perturbation signal that locates the gold nanoparticle is no more than one order of magnitude below the backscattering of the isolated microsphere, even though the nanoparticle is only $1 / 100$ th the diameter of the microsphere. 
In combination, References 2 and ${ }^{11}$ indicate that it is possible to use visible light to detect, size, and locate with subdiffraction resolution nanoparticles as small as a few nanometers at standoff distances of appreciable fractions of the wavelength. A potential biophotonics application involves the detection of nanoparticles attached to the membranes of living cells in an aqueous environment. In a conceptual experiment, a cell could be scanned with the nanojet emanating from an optically trapped microsphere, which would have a soft-spring recoil and hence would minimize potential damage to the biological sample.

\section{Directional Emission in a Symmetrical Aggregate of Spherical Particles}

Gerlach et al. ${ }^{12}$ reported optical measurements of small symmetrical arrays of contacting latex microspheres (refractive index $n=1.68$; diameter $=5.37 \pm 0.07 \mu \mathrm{m}$, i.e., $\sim 16 \lambda$ ). Here, strong optical coupling between the microspheres can result in a complex rearrangement of their mode structure similar to the electronic molecular orbitals in a chemical molecule. This analogy motivated the usage of the term "photonic molecule." 12

The following compact groupings were tested: (a) three microspheres located at the vertices of an equilateral triangle; (b) five microspheres located at the vertices of an equilateral pentagon; and (c) seven microspheres, with six located at the vertices of an equilateral hexagon surrounding a seventh microsphere located at the center. Each grouping was formed on a silicon wafer substrate. Upon illumination with a defocused laser beam perpendicular to the silicon substrate (i.e., the molecule plane), photonic nanojets were observed propagating outward symmetrically from each of the microspheres located at the vertices of each grouping. Nanojet emission was visible in the far field due to reflection from the silicon substrate.

It was concluded that the observed directed emission of photonic nanojets enables possible uses of symmetrical groupings of microspheres as transverse beam splitters having a number of output ports equal to the number of microspheres located at the vertices of the equilateral triangle, pentagon, hexagon, etc. arrangement being used. Furthermore, such beam splitting could allow transverse coupling from an impinging laser beam onto photonic components such as waveguides laid out along a planar substrate. This coupling could be possible with subwavelength spatial accuracy, and yield much higher intensities than conventional subwavelength coupling techniques using scanning near-field optical microscopy tips.

\section{Inspection of Semiconductor Wafers}

Chen et al. ${ }^{17}$ patented means to perform optical metrology of a semiconductor wafer using a photonic nanojet. In this technique, the inspection area of the wafer is scanned with a nanojet, and measurements are obtained of the retroreflected light from the dielectric microsphere used to generate the nanojet. The presence, type, and dimensions of a wafer-surface feature are determined by comparing the backscattered light signature with a library of such signatures for both desired structures and contaminating particles.

\section{Optical Trapping of Metal Nanoparticles}

Cui et al. ${ }^{18}$ theoretically investigated the optical forces acting on a metal nanoparticle located within a photonic nanojet generated by a plane-wave illuminated dielectric microcylinder. It was found that these optical forces depend strongly on the dielectric permittivity of the nanoparticle and the intensity and beamwidth of the nanojet. While subwavelength metal nanoparticles can be efficiently trapped within a nanojet, the attractive force can be changed to a repulsive force simply by varying the polarization of the incident illumination. This reversal is related to the nanoparticle's polarizability and excitation of localized surface plasmons. Overall, these findings are promising for the application of photonic nanojets to "provide highly confined force fields to efficiently organize nanostructures in the nanoscale." 18 


\section{Ultrahigh-Density Optical Data-Storage Disks}

Kong et al. ${ }^{14,} 19$ computationally and experimentally investigated the potential application of the photonic nanojet to implement ultrahigh-density optical data-storage disks wherein data are encoded as nanoscale pits in a metal substrate. This application is based upon the giant backscattering perturbation phenomenon previously reported for isolated nanoparticles located within a nanojet; ${ }^{2,5,6}$ but here nanoscale pits in the disk substrate (rather than isolated nanoparticles) generate the backscattering perturbations. As illustrated in Fig. 10, microwave measurements on dimensionally scaled-up disk models and FDTD computational models indicate that pits having a lateral area of 0.025 square wavelengths, i.e., much smaller than current BluRay ${ }^{\mathrm{TM}}$ device features, can be robustly detected with a contrast ratio approximately $28 \mathrm{~dB}$ greater than provided by a lens system. ${ }^{14,} 26$ These findings, subsequently extended to optical wavelengths ${ }^{19}$ using FDTD computational modeling, suggest that photonic nanojets could lead to the development of optical-disk data-storage technology having much greater capacity and speed than the present state of the art.

\section{Summary and Conclusions}

This paper has reviewed the substantial body of literature emerging since 2004 concerning photonic nanojets. Discussions included a review of the initial identification of the photonic nanojet for the dielectric cylinder and sphere, properties of the photonic nanojet, nanojet theory, laboratory observations, and potential applications. Photonic nanojets exhibit a unique combination of desirable attributes including wave propagation (rather than evanescence) along multiwavelength paths; narrow, potentially subdiffraction beamwidths; high intensity; little wavelength sensitivity; and giant backscattering perturbations for nanoparticles and nanopits. Consequently, photonic nanojets hold considerable promise for advancing current nanophotonic technologies ranging from ultramicroscopy to optical data storage to nanopatterning to biophotonics.

\section{Acknowledgments}

The photonic nanojet research by our group was supported in part by National Institutes of Health Grant R01 EB003682 and National Science Foundation Grants CBET-0939778 and CBET-0522639. Alexander Heifetz, Ph.D. was supported by the Canary Foundation/American Cancer Society Early Detection Postdoctoral Fellowship. Jim Spadaro and Nikola Borisov managed and maintained Prof. Backman's computer cluster.

\section{References}

1. Chen ZG, Taflove A, Backman V. Optics Exp 2004;12:1214.

2. Li X, Chen Z, Taflove A, Backman V. Optics Exp 2005;13:526.

3. Lecler S, Takakura Y, Meyrueis P. Optics Lett 2005;30:2641.

4. Itagi AV, Challener WA. J Opt Soc America A 2005;22:2847.

5. Chen ZG, Li X, Taflove A, Backman V. Optics Lett 2006;31:196.

6. Heifetz A, Huang K, Sahakian AV, Li X, Taflove A, Backman V. Appl Phys Lett 2006;89:221118.

7. Kapitonov AM, Astratov VN. Optics Lett 2007;32:409.

8. Yi KJ, Wang H, Lu YF, Yang ZY. J Applied Physics 2007;101:063528.

9. Lecler S, Haacke S, Lecong N, Crégut O, Rehspringer JL, Hirlimann C. Optics Exp 2007;15:4935.

10. Wu W, Katsnelson A, Memis OG, Mohseni H. Nanotechnology 2007;18:485302.

11. Heifetz A, Simpson JJ, Kong SC, Taflove A, Backman V. Optics Exp 2007;15:17334.

12. Gerlach M, Rakovich YP, Donegan JF. Optics Exp 2007;15:17343.

13. Ferrand P, Wenger J, Devilez A, Pianta M, Stout B, Bonod N, Popov E, Rigneault H. Optics Exp 2008;16:6930.

14. Kong SC, Sahakian AV, Heifetz A, Taflove A, Backman V. Appl Phys Lett 2008;92:211102. 
15. Yang S, Astratov VN. Appl Phys Lett 2008;92:261111.

16. McLeod E, Arnold CB. Nature Nanotech 2008;3:413.

17. Chen Z, Chu H, Li S. U.S Patent 2008;7:394, 535.

18. Cui X, Erni D, Hafner C. Optics Exp 2008;16:13560.

19. Kong SC, Sahakian A, Taflove A, Backman V. Optics Exp 2008;16:13713.

20. Devilez A, Stout B, Bonod N, Popov E. Optics Exp 2008;16:14200.

21. Taflove, A.; Hagness, SC. Computational Electrodynamics: The Finite-Difference Time-Domain Method. Vol. 3. Artech; Boston: 2005.

22. Adler CL, Lock JA, Stone BR, Garcia CJ. J Opt Soc America A 1997;14:1305.

23. Lock JA, Adler CL, Hovenac EA. J Opt Soc America A 2000;17:1846.

24. Xu YL, Wang RT. Phys Rev E 1998;58:3931.

25. Online: http://www.astro.ufl.edu/ Xu/

26. Veerman JAC, Wachters AJH, van der Lee AM, Urbach HP. Optics Exp 2007;15:2075. 


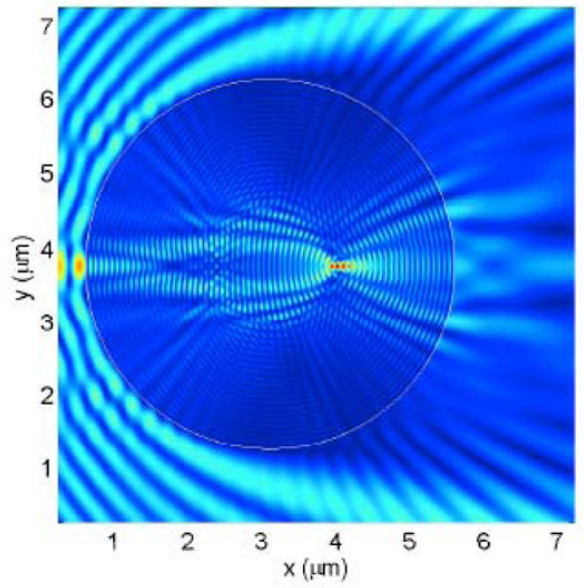

(a)
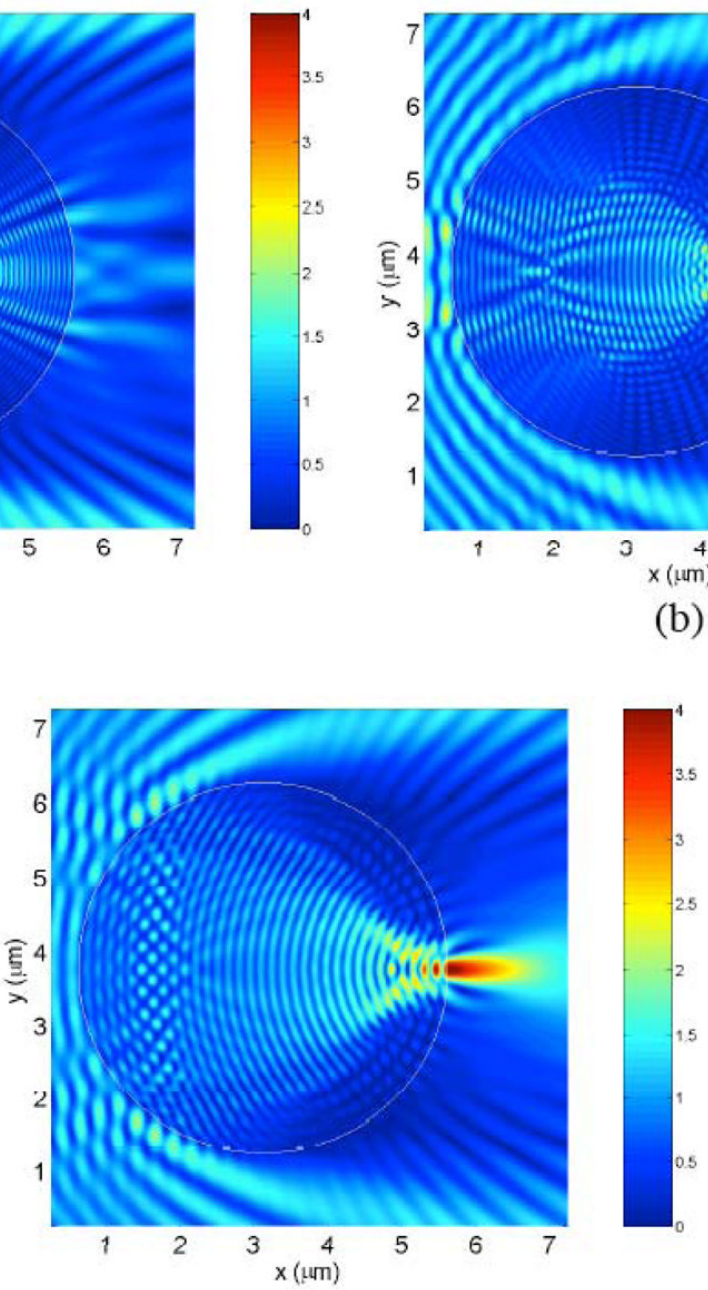

(c)

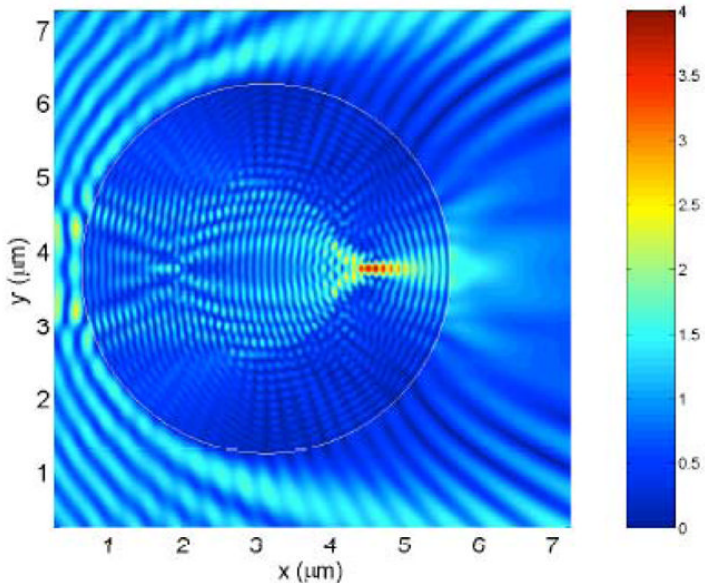

(b)

Fig. 1.

Visualizations of the evolution of a photonic nanojet as the refractive index of a plane-waveilluminated circular dielectric cylinder decreases. The FDTD-computed envelope of the sinusoidal steady-state electric field is visualized for a $d=5 \mu \mathrm{m}$ diameter cylinder of uniform refractive index $n_{1}$ embedded within an infinite vacuum medium of refractive index $n_{2}=1.0$. Light of wavelength $\lambda_{2}=500 \mathrm{~nm}$ propagates from left to right in medium 2. (a) $n_{1}=3.5$; (b) $n_{1}=2.5$; (c) $n_{1}=1.7$. Reprinted with permission from Chen, Taflove, and Backman, Optics Express 12(7):1214-1220, April 5, 2004. Copyright 2004, Optical Society of America. 


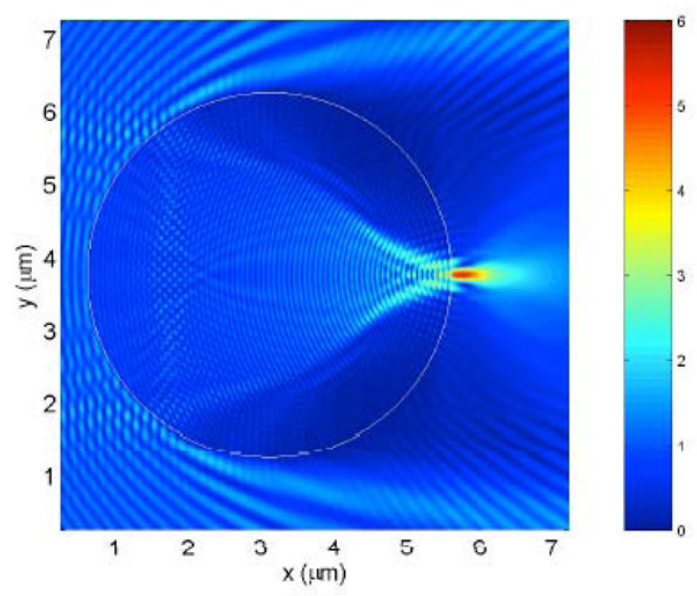

(a)

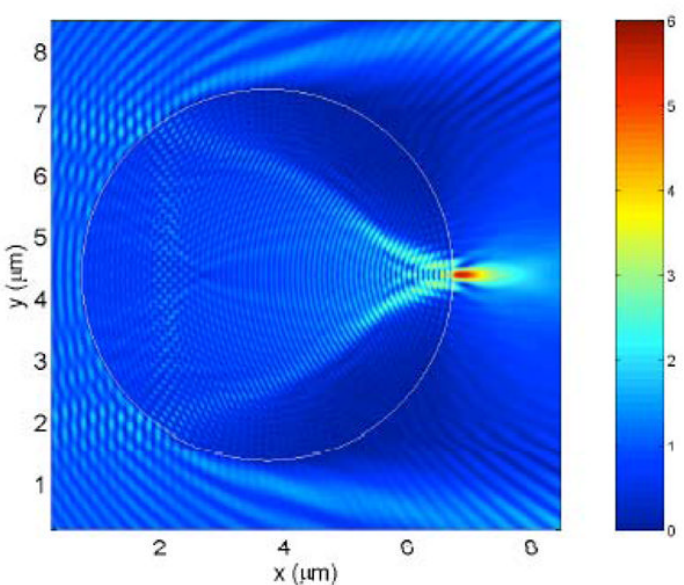

(b)

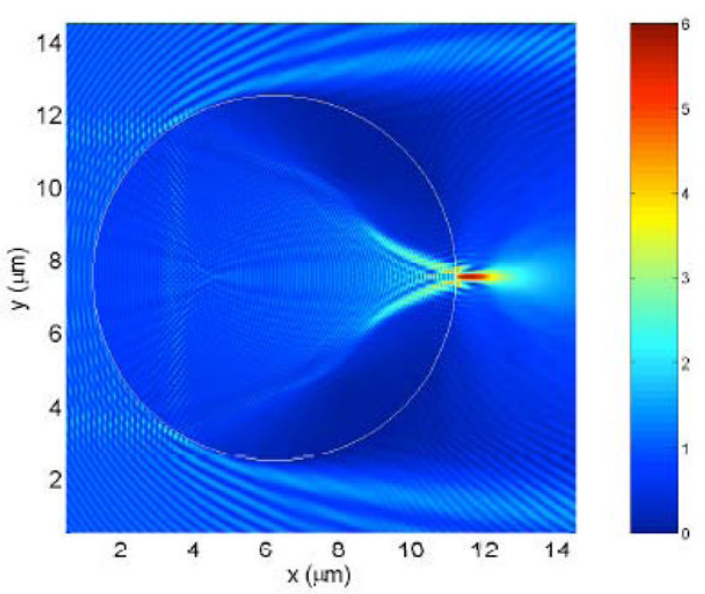

(c)

Fig. 2.

Visualizations of the generation of photonic nanojets similar to that in Fig. 1(c) for three different combinations of $d, n_{1}, n_{2}$, and $\lambda_{2}$. (a) $d=5 \mu \mathrm{m}, n_{1}=3.5, n_{2}=2.0, \lambda_{2}=250 \mathrm{~nm}$; (b) $d=6 \mu \mathrm{m}, n_{1}=2.3275, n_{2}=1.33, \lambda_{2}=300 \mathrm{~nm}$; (c) $d=10 \mu \mathrm{m}, n_{1}=2.3275, n_{2}=1.33, \lambda_{2}=300$ nm. Reprinted with permission from Chen, Taflove, and Backman, Optics Express 12(7):12141220, April 5, 2004. Copyright 2004, Optical Society of America. 
(a)

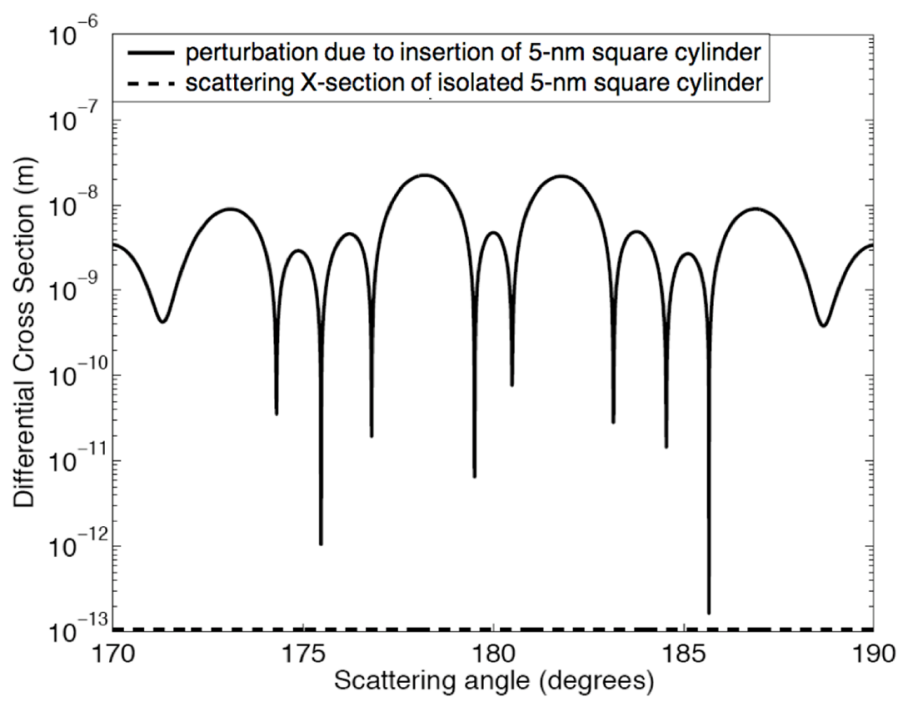

(b)

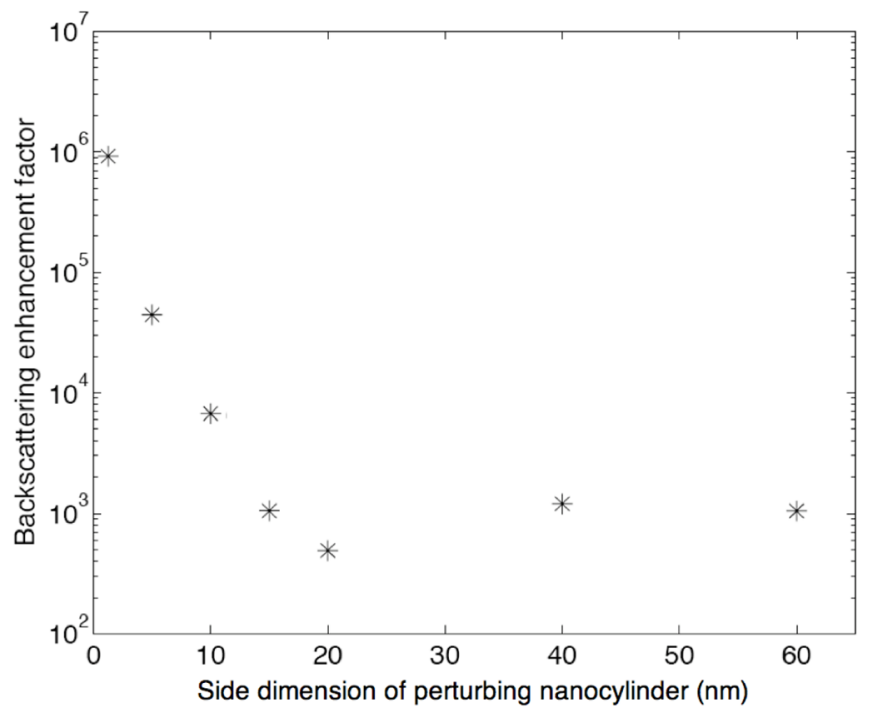

Fig. 3.

(a) FDTD-calculated absolute value of the perturbation of the differential scattering crosssection of the circular (primary) dielectric cylinder for the case of Fig. 2(b) $\left(d_{\mu}=6 \mu \mathrm{m}, n_{1}=\right.$ $2.3275, n_{2}=1.33, \lambda_{2}=300 \mathrm{~nm}$ ). The perturbation is caused by placing a $5-\mathrm{nm}$ square dielectric nanocylinder $\left(n_{v}=1.5\right)$ on the surface of the primary microcylinder at the center of the photonic nanojet. (b) Effective backscattering enhancement factor $E$ vs. side dimension of the perturbing nanocylinder. Reprinted with permission from Chen, Taflove, and Backman, Optics Express 12 (7):1214-1220, April 5, 2004. Copyright 2004, Optical Society of America. 

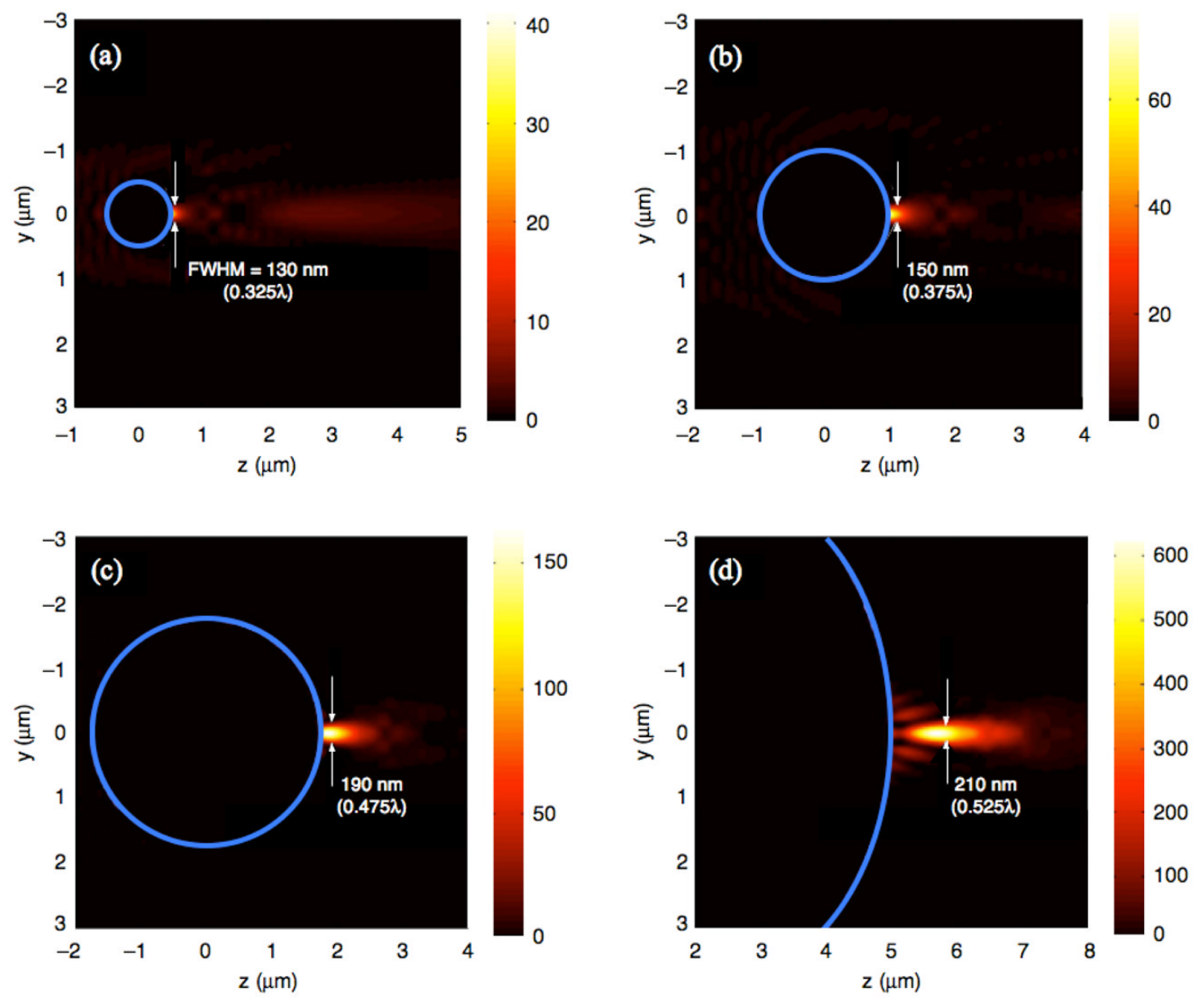

Fig. 4.

Visualizations of the photonic nanojets generated by illuminating dielectric microspheres of refractive index $n_{\mu}=1.59$ in vacuum with a $\lambda=400 \mathrm{~nm}, x$-polarized, $z$-propagating plane waves of unit intensity. The near fields are computed using the Mie series. (a) $d_{\mu}=1 \mu \mathrm{m}$ microsphere diameter; (b) $d_{\mu}=2 \mu \mathrm{m}$; (c) $d_{\mu}=3.5 \mu \mathrm{m}$; (d) $d_{\mu}=8 \mu \mathrm{m}$. Reprinted with permission from $\mathrm{Li}$, Chen, Taflove, and Backman, Optics Express 13(2):526-533, Jan. 24, 2005. Copyright 2005, Optical Society of America. 

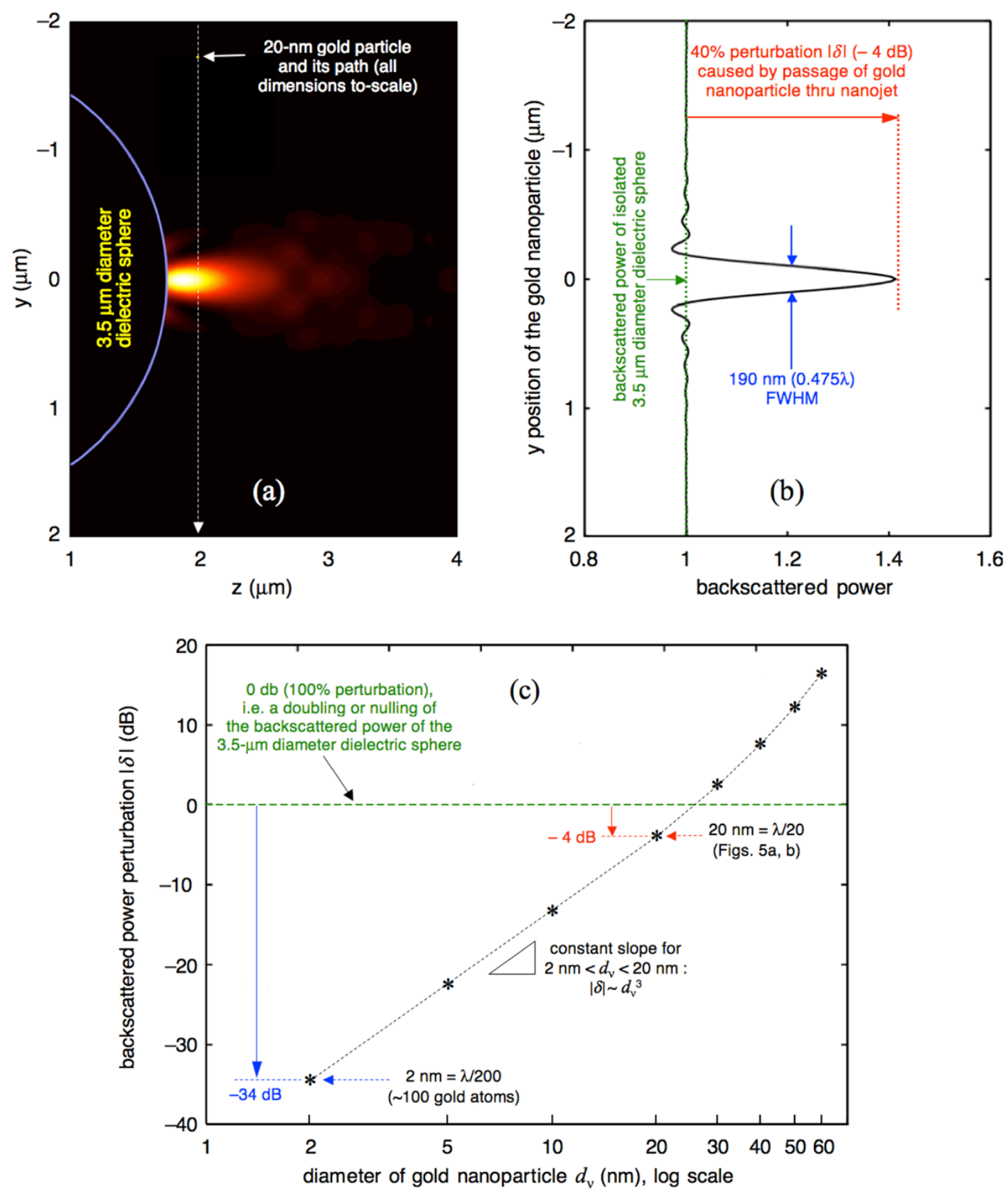

Fig. 5.

(a) Passage of a $d_{v}=20 \mathrm{~nm}$ diameter gold nanoparticle through the nanojet of Fig. 4(c). (b) Resulting $40 \%$ perturbation $|\delta|$ of the backscattered power of the $3.5-\mu \mathrm{m}$ diameter dielectric microsphere. (c) Backscattered power perturbation $|\delta|$ in $\mathrm{dB}$ relative to the backscattered power of the isolated 3.5- $\mu \mathrm{m}$ diameter dielectric microsphere of (a). Reprinted with permission from Li, Chen, Taflove, and Backman, Optics Express 13(2):526-533, Jan. 24, 2005. Copyright 2005, Optical Society of America. 


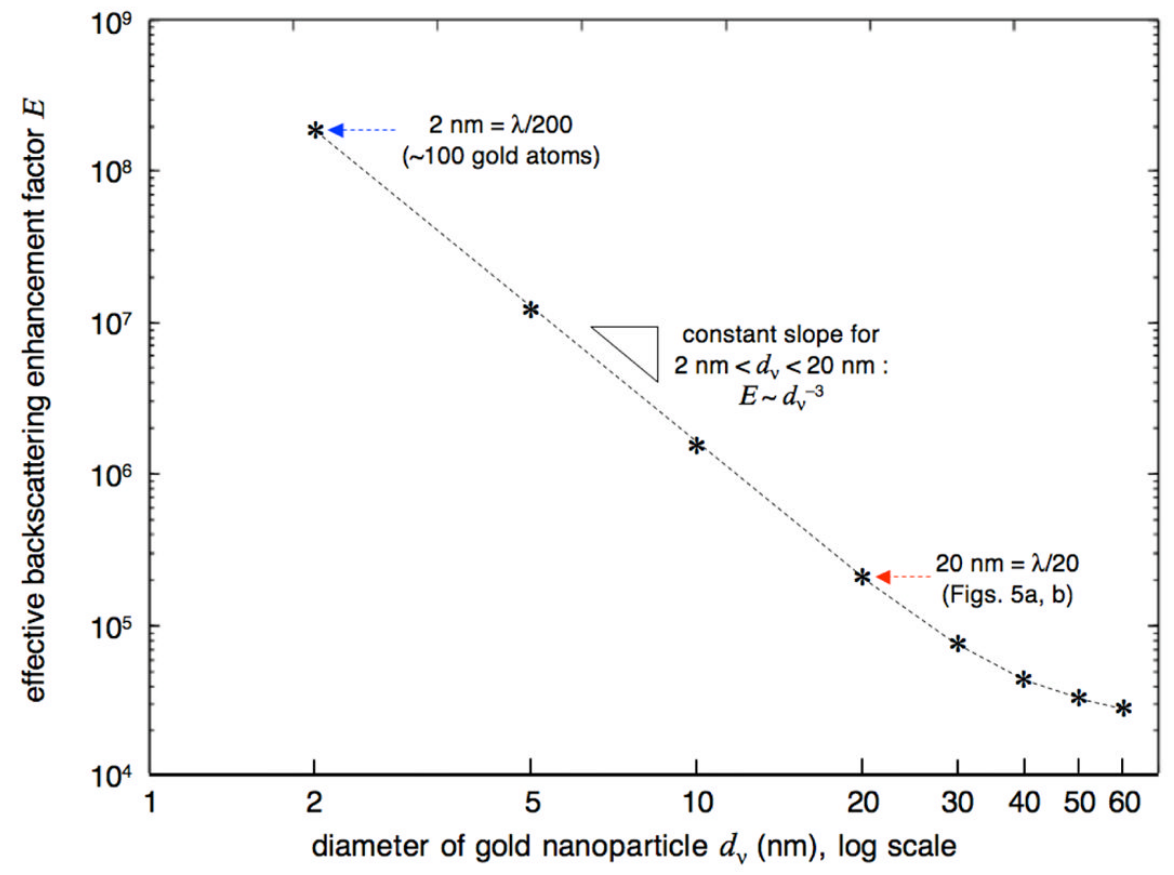

(a)

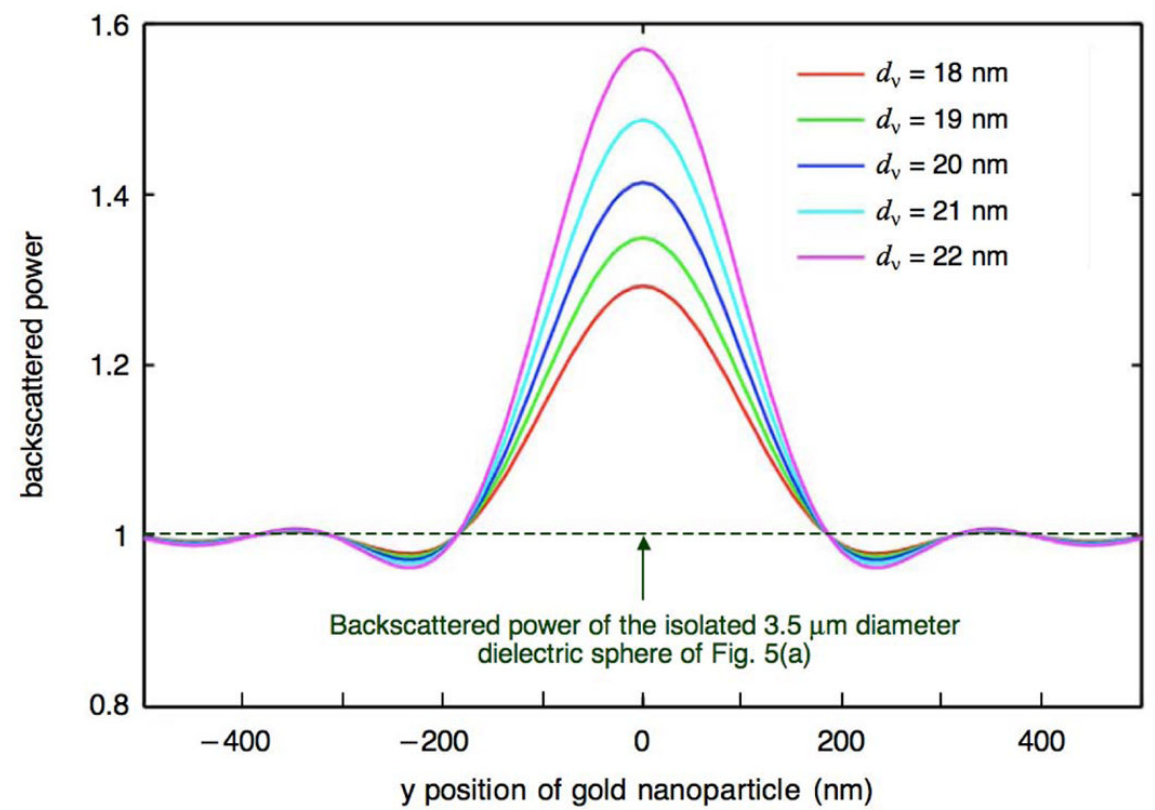

(b)

Fig. 6.

(a) Effective backscattering enhancement factor $E$ of the perturbing gold nanoparticle vs. its diameter $d_{v}$. (b) Sensitivity of the backscattered power of the microsphere/gold nanoparticle system to nanometer variations of the gold nanoparticle diameter $d_{v}$ from 18 to $22 \mathrm{~nm}$.

Reprinted with permission from Li, Chen, Taflove, and Backman, Optics Express 13(2):526533, Jan. 24, 2005. Copyright 2005, Optical Society of America. 


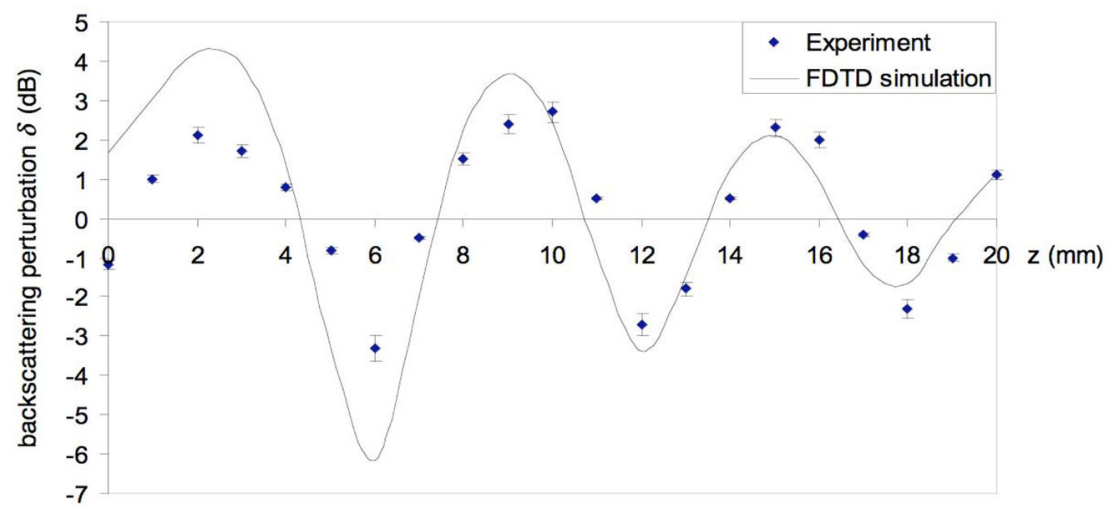

(a)

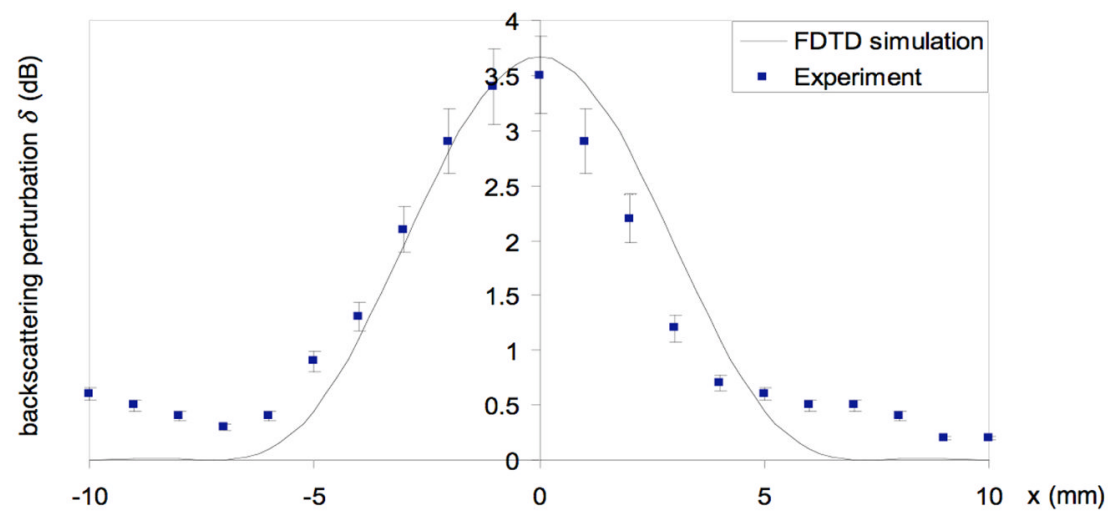

(b)

Fig. 7.

(a) Measured and FDTD-calculated backscattering perturbation $\delta(\mathrm{dB})$ caused by a $d_{v}=1 \mathrm{~mm}$ $(\lambda / 10)$ metal particle scanned along the central propagation axis of the $30-\mathrm{GHz}(\lambda=1 \mathrm{~cm})$ microwave jet. $z$ is the distance between the shadow-side surface of the $d_{\mu}=7.62 \mathrm{~cm}(7.62 \lambda)$ acrylic sphere and the surface of the metal particle. (b) Same as (a), but for the 1-mm metal particle scanned laterally across the microwave jet in the electric-field direction along a line at $z=9 \mathrm{~mm}(0.9 \lambda)$ from the shadow-side surface of the acrylic sphere. Reprinted with permission from A. Heifetz, K. Huang, A. V. Sahakian, X. Li, A. Taflove, and V. Backman, Applied Physics Letters 89(22): 221118, Nov. 27, 2006. Copyright 2006, American Institute of Physics. 


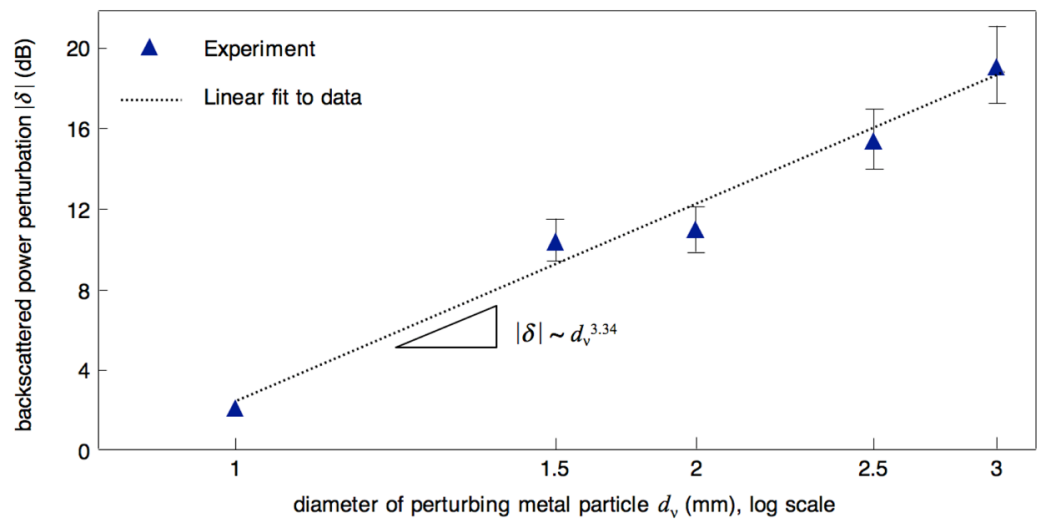

Fig. 8.

Measured dependence of the backscattered power perturbation $|\delta|(\mathrm{dB})$ upon the diameter $d_{v}$ of the perturbing metal particle that is located on the central propagation axis of the $30-\mathrm{GHz}$ $(\lambda=1 \mathrm{~cm})$ microwave jet at the distance $z=9 \mathrm{~mm}(0.9 \lambda)$ from the shadow-side surface of the $d_{\mu}=7.62 \mathrm{~cm}(7.62 \lambda)$ acrylic sphere. Reprinted with permission from A. Heifetz, K. Huang, A. V. Sahakian, X. Li, A. Taflove, and V. Backman, Applied Physics Letters 89(22): 221118, Nov. 27, 2006. Copyright 2006, American Institute of Physics. 

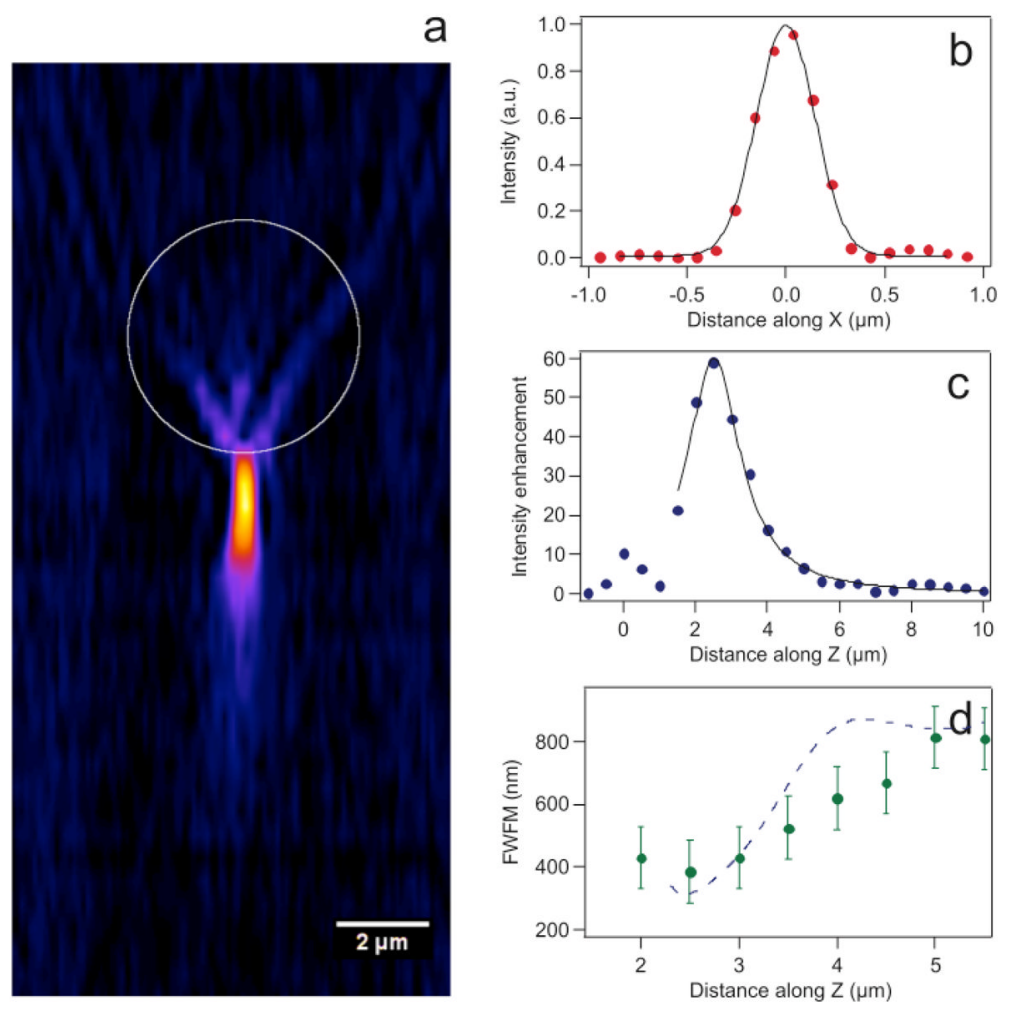

Fig. 9.

(a) Visualization of the experimentally measured photonic nanojet generated by a $5 \mu \mathrm{m}$ latex microsphere (refractive index $=1.6$ ) resting on a glass coverslip, as viewed in a vertical plane cutting through the center of the microsphere. The illumination is a downward-propagating plane wave at $\lambda=520 \mathrm{~nm}$. (b) Measured intensity normalized to a peak value of unity along a horizontal line cutting through the narrowest point of the nanojet. Red dots: measured data; solid line: Gaussian curve fit. (c) Measured intensity normalized to that of the incident wave along a vertical line below the microsphere at the center of the nanojet. Blue dots: measured data; solid line: Lorenzian curve fit. (d) FWHM beamwidth of the nanojet vs. distance below the microsphere. Green dots: measured data; dashed line: numerical simulation results for the microsphere assumed to be in free space. Reprinted with permission from Ferrand, Wenger, Devilez, Pianta, Stout, Bonod, Popov, and Rigneault, Optics Express 16(10): 6930-6940, May 12, 2008. Copyright 2008, Optical Society of America. 


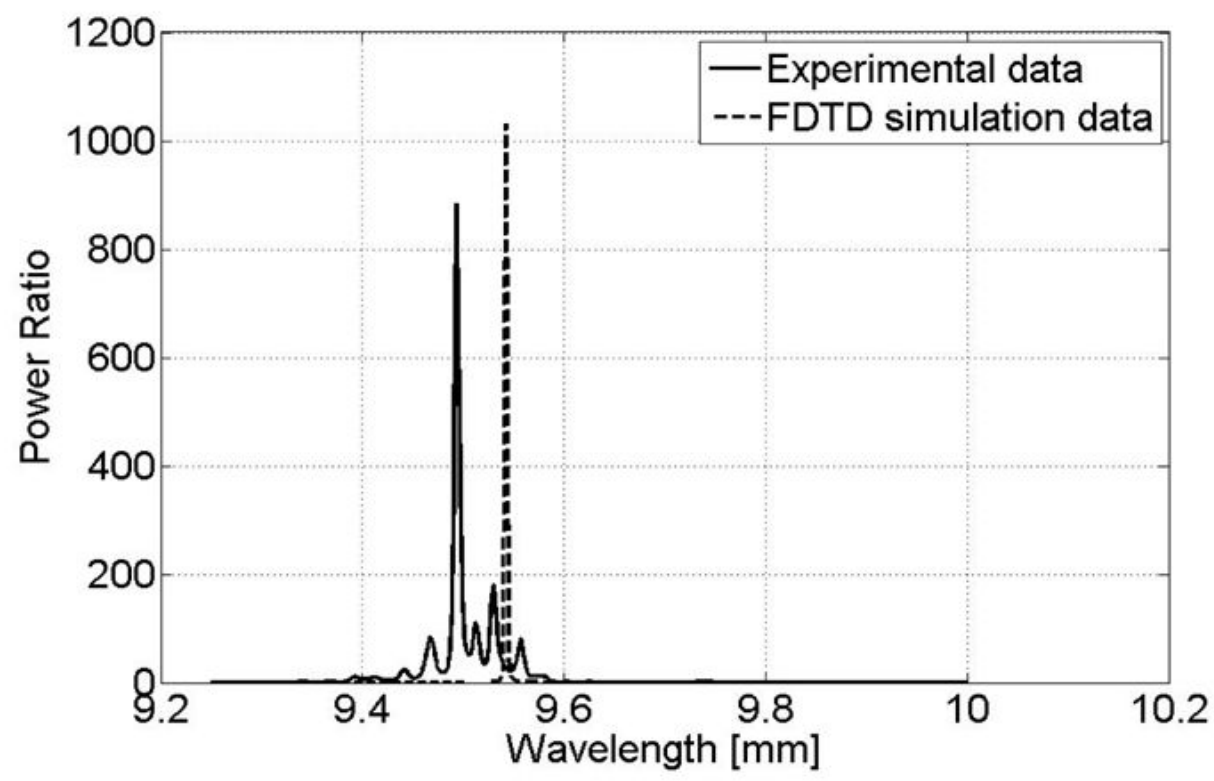

(b)

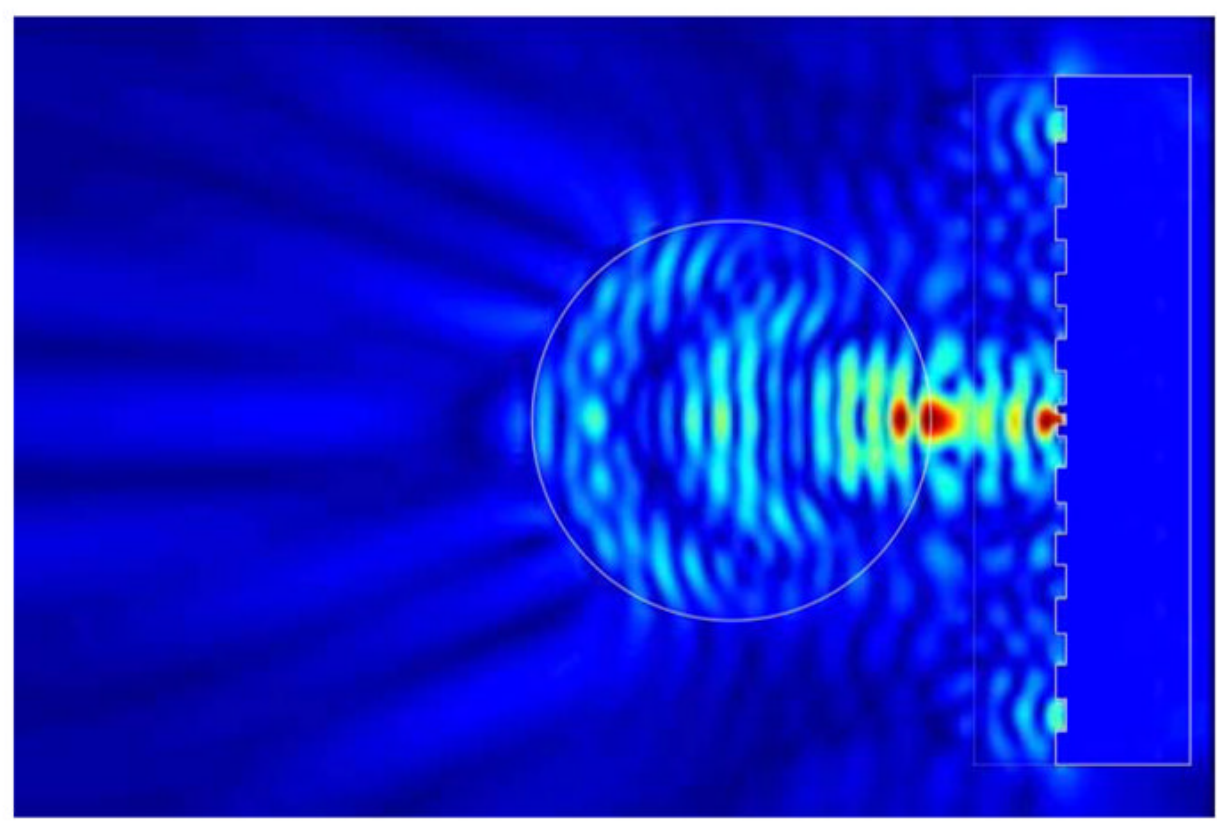

Fig. 10.

(a) Comparative experimental and FDTD data for the nanojet-induced no-pit-to-pit power ratio versus wavelength for a rectangular pit of lateral dimensions $1.25 \mathrm{~mm}\left(\lambda_{0} / 8\right) \times 2 \mathrm{~mm}\left(\lambda_{0} / 5\right)$ and depth $2 \mathrm{~mm}\left(\lambda_{0} / 5\right)$. Both datasets show a peak no-pit-to-pit power ratio that is approximately 700-times (i.e., $28 \mathrm{~dB}$ ) greater than the 1.2 value reported in Reference 25 using a conventional lens for an octagonal pit of approximately the same lateral area in square wavelengths. (b) Visualization of the FDTD-computed microwave intensity distribution for the pit case of (a). There is a strong nanojet intensity localization in the vicinity of the pit with a peak value of $\sim 225$ times the incident. The backscattered wave intensity decreases with distance from the front surface of the sphere faster than for the no-pit case, yielding a much 
weaker far-field backscattered response. Reprinted with permission from S.-C. Kong, A. V. Sahakian, A. Heifetz, A. Taflove, and V. Backman, Applied Physics Letters 92(21): 211102, May 26, 2008. Copyright 2008, American Institute of Physics. 